PREPARED FOR SUBMISSION TO JCAP

UMD-PP-10-019

\title{
The Consistency of Fermi-LAT Observations of the Galactic Center with a Millisecond Pulsar Population in the Central Stellar Cluster
}

\section{Kevork N. Abazajian}

Maryland Center for Fundamental Physics \& Joint Space-Science Institute, Department of Physics, University of Maryland, College Park, Maryland 20742 USA

E-mail: kev@umd.edu

\begin{abstract}
I show that the spectrum and morphology of a recent Fermi-LAT observation of the Galaxy center are consistent with a millisecond pulsar population in the nuclear Central stellar cluster of the Milky Way. The Galaxy Center gamma-ray spectrum is consistent with the spectrum of four of eight globular clusters that have been detected in the gamma-ray. A dark matter annihilation interpretation cannot be ruled out, though no unique features exist that would require this conclusion.
\end{abstract}

Keywords: millisecond pulsars, gamma ray experiments, dark matter theory

ArXiv ePrint: arXiv:1011.4275

\section{Introduction}

The ability of gamma-ray observatories to provide a window on dark matter annihilation signals has been known for some time, (e.g. [1]). The recent manuscript by Hooper \& Goodenough [2] performs a detailed analysis to extract the signal in the gamma-ray of the dynamical center of our Milky Way Galaxy as observed by the Large Area Telescope (LAT) aboard the Fermi Gamma-Ray Space Telescope. That work interpreted the morphology and spectral feature of the signal within the inner $1.25^{\circ}$ (175 parsec radius) from the Galactic dynamic center to be inconsistent with any known astrophysical sources, and further claimed for it to be accounted for by the presence of annihilating light $(\sim 8 \mathrm{GeV})$ dark matter. In this short note, I show that the signal as seen by Hooper \& Goodenough (hereafter HG) is from the Galactic Central stellar cluster and is consistent with prior observations of gamma-ray emission from massive stellar globular clusters with a population of millisecond pulsars (MSP) $[3]$. 


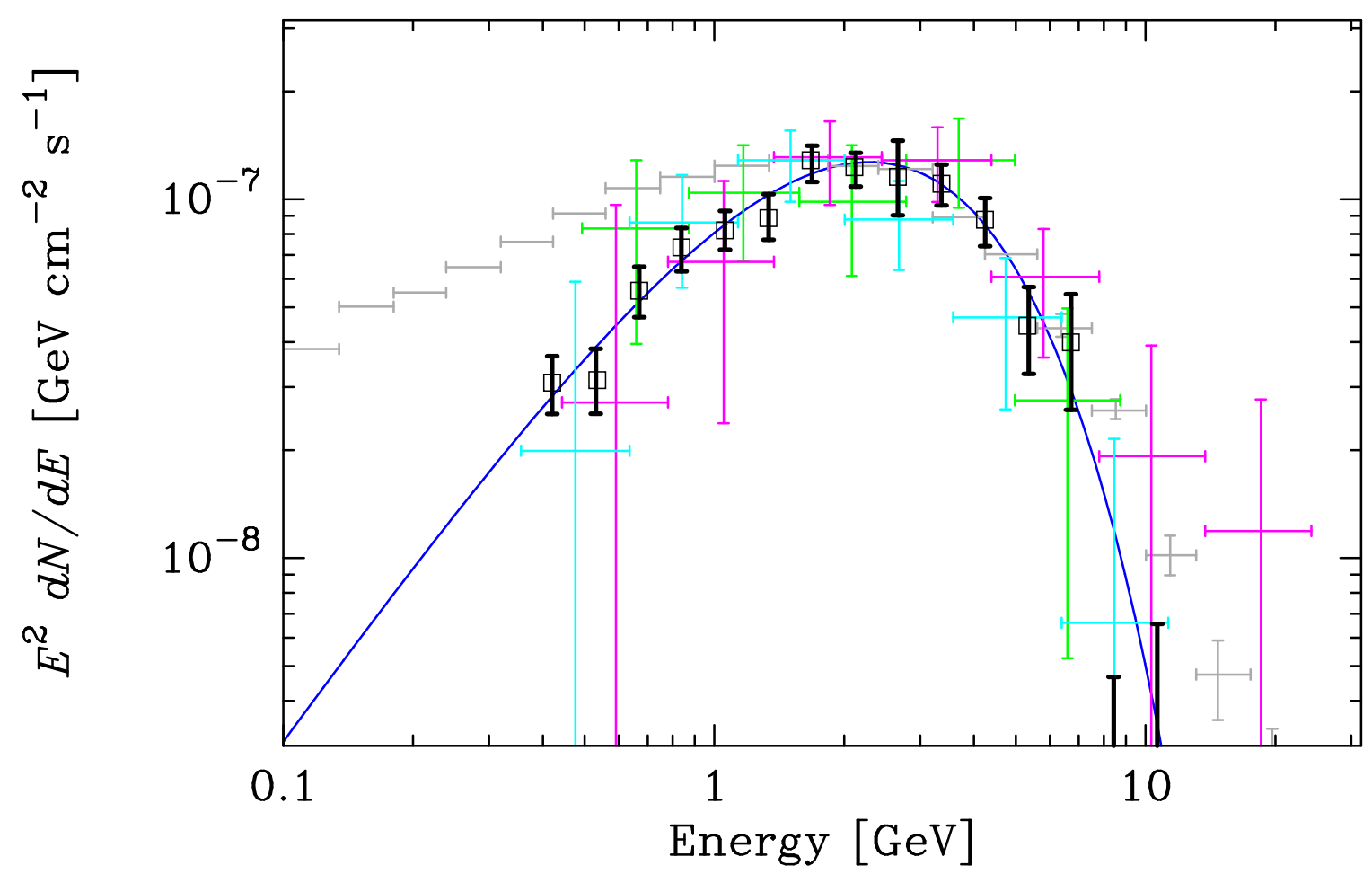

Figure 1. Shown is the spectrum $E^{2} d N / d E$ of the HG source (thick black points with errors), and its best fit power law with exponential cutoff curve (blue). The HG spectrum power-law index is consistent with the globular cluster gamma ray emission of Omega Cen (green), NGC 6388 (cyan) and M 28 (magenta) [3]. The spectrum of the Geminga pulsar (light grey) [4] has a nearly identical peak energy and exponential cutoff and a softer power-law index than the HG source. The spectra of Omega Cen and M 28 are shifted by +0.14 dex and +0.34 dex in energy, respectively, to illustrate the consistency in power-law index. NGC 6388 is not shifted in energy. The HG source is consistent, within errors, with the shape of the intrinsic spectrum of all the plotted globular cluster sources. All sources are normalized to the HG peak flux.

\section{Spectrum}

In general, pulsar gamma-ray spectra have a flux peak at $\sim 2-3 \mathrm{GeV}$ and a spectrum $d N / d E \propto E^{-\Gamma} \exp \left(E / E_{\mathrm{c}}\right)$ with a power law with index $\Gamma$ in the range 0.5-2.5 and exponential cutoff energy $E_{\mathrm{c}}$ at 1-5 GeV, a range consistent with the HG source [5]. Note that HG compare to the average millisecond pulsar spectrum and find it is not consistent, and then reach the conclusion that no astrophysical source can produce the HG spectrum. However, this is not appropriate, but rather consistency with any set of pulsars or stellar cluster population of pulsars must be ruled out before claiming inconsistency with astrophysical sources.

The spectrum of the HG source can be well fit by a pulsar-like power law plus exponential cutoff spectrum. The spectrum below $7 \mathrm{GeV}$ is well fit by $d N / d E \propto E^{-\Gamma} \exp \left(E / E_{\mathrm{c}}\right)$, with $\Gamma=0.29 \pm 0.12 \mathrm{GeV}$ and $E_{\mathrm{c}}=1.34 \pm 0.12 \mathrm{GeV}$ and a reduced $\chi^{2} / \mathrm{DOF}=0.5$. The spectrum and best fit curve is shown in Fig. (1).

Several pulsars in the First Fermi-LAT Catalog of Gamma-ray Pulsars [5], including $\mathrm{J} 1958+2846$, J2032+4127 and J2043+2740, have a power-law index and exponential cutoff consistent with the HG source. The Geminga pulsar has a detailed published gamma-ray spectrum which has a similar spectral peak, $2-3 \mathrm{GeV}$ and cutoff as the HG source [4]. The 
Geminga spectrum is shown for comparison in Fig. (1). However, Geminga has a shallower power-law spectral index $\Gamma=1.08 \pm 0.02$.

However, it is not expected that the extreme density of the Galactic Central stellar cluster harbors only a single pulsar. The Central stellar cluster (nuclear bulge) of the Milky Way is coincident with the observational region of HG central spectral signal, and extends across the full $\sim 1^{\circ}$ from the dynamical center (or $\sim 300 \mathrm{pc}$ across) of that spectral analysis region. The spectrum of the HG Central stellar cluster region $\left(\Gamma=0.29 \pm 0.12, E_{\mathrm{c}}=\right.$ $1.34 \pm 0.12$ ) is consistent with four of the eight globular clusters that have been observed by Fermi-LAT and seen to have gamma-ray emission [3]:

$$
\begin{aligned}
\text { Omega Cen: } & \Gamma=0.7_{-0.6-0.4}^{+0.7+0.4}, E_{\mathrm{c}}=1.2_{-0.4-0.2}^{+0.7+0.2}, \\
\text { NGC 6388: } & \Gamma=1.1_{-0.5-0.8}^{+0.7+0.8}, E_{\mathrm{c}}=1.8_{-0.7-0.6}^{+1.2+1.8}, \\
\text { M 28: } & \Gamma=1.1_{-0.5-0.7}^{+0.7+0.6}, E_{\mathrm{c}}=1.0_{-0.3-0.2}^{+0.6+0.4}, \\
\text { NGC 6652: } & \Gamma=1.0_{-0.5-0.3}^{+0.6+0.3}, E_{\mathrm{c}}=1.8_{-0.6-0.3}^{+1.2+0.4} .
\end{aligned}
$$

Three of these spectra are shown in Fig. (1). Note that a direct quantitative comparison of the fit to the HG source spectrum presented above with the globular cluster fits, Eqs. (2.1), cannot be rigorously performed since the sets of fits use different methods. The fit performed in this analysis is to the points and errors presented in HG, while the fits in Eqs. (2.1) from Ref. [3] are performed with the Fermi-LAT analysis tool gtlike, which bins the spectrum differently and more accurately incorporates the photon statistics. The fit between HG and the globular clusters are obviously qualitatively consistent (Fig. 1), but a detailed statistical comparison can only be established with gtlike analyses of all regions. Most importantly, over-subtraction or under-subtraction of the diffuse component of an observation can lead to a systematic uncertainty that must also be included in a full error analysis of the Galaxy Central stellar cluster spectrum [6]. This was not performed in the HG analysis. Oversubtraction of the diffuse component may lead to a high estimate of the intrinsic spectral index $\Gamma$.

\section{Morphology and Luminosity}

The Central stellar cluster of the Milky Way has a stellar mass of approximately $4 \times 10^{9} M_{\odot}[7]$ and harbors $2357 \mathrm{X}$-ray point sources within the central $17^{\prime} \times 17^{\prime}$ alone [8]. In comparison, Omega Cen is the most massive stellar globular cluster in our Galaxy's halo with mass $5 \times 10^{6} M_{\odot}[9]$, and has 45-70 X-ray sources associated with it [10].

The peak energy flux in gamma rays of the Central stellar cluster associated with the HG source is approximately 200 times that of Omega Cen, when corrected for distance. Given the three order of magnitude greater mass scale of the Central Galactic stellar cluster and its relative high-energy activity to that of the globular clusters, the factor of $\sim 200$ enhancement of flux is not unreasonable.

In the same regard, the gamma ray profile of a globular cluster like Omega Cen only extends to $7.5^{\prime}$, while that observed in the Central stellar cluster by HG is approximately

$30^{\prime}$, which can also be accounted for by the 3 order of magnitude larger mass scale of the Central stellar cluster. 


\section{Conclusions}

The gamma-ray flux spectrum and profile observed by HG towards the Central stellar cluster of the Milky Way is consistent with other stellar cluster populations in observed globular clusters. The spectral index, exponential cutoff and peak flux energy is consistent with four of the eight detected globular clusters in the gamma-ray by Fermi-LAT. Therefore, there exists is no feature of the HG spectral source that necessarily requires a dark matter annihilation interpretation.

Of the detected gamma-ray observed globular clusters, only 5 of 8 harbor detected MSPs [3]. The gamma-ray emission from stellar clusters such as the Central stellar cluster as well as globular clusters is expected to be dominated by MSP emission due to enhanced binary formation in these systems, where MSPs are spun up by accretion from their binary partners. Therefore, it is likely that the detection of the peaked spectrum towards the Galactic Central stellar cluster by Hooper \& Goodenough is that of a population of millisecond pulsars bound within this massive stellar cluster. Obscuration, crowding, and absorption towards the central region of the Milky Way makes direct detection of the MSPs difficult, though this identification of the spectral signature of stellar cluster-like MSPs in the Galactic Center may motivate deeper searches.

Acknowledgments - I would like to thank Prateek Agrawal, Steve Blanchet, Z. Chacko, J. Pat Harding, Manoj Kaplinghat, Jürgen Knödlseder, Julie McEnery, and Paul Ray for useful discussions. In particular, I would like to thank Elizabeth Ferrara for discussions and detailed comments on the manuscript, as well as her and Chris Shrader for organizing a GSFC Fermi-LAT Science Workshop on November 16, 2010 which led to this analysis. K.A. is supported by NSF Grant 07-57966 and NSF CAREER Award 09-55415.

\section{References}

[1] G. Jungman, M. Kamionkowski, and K. Griest, Supersymmetric dark matter, Phys. Rept. 267 (1996) 195-373, [hep-ph/9506380].

[2] D. Hooper and L. Goodenough, Dark Matter Annihilation in The Galactic Center As Seen by the Fermi Gamma Ray Space Telescope, arXiv:1010.2752.

[3] Fermi-LAT Collaboration, A. Abdo et. al., A population of gamma-ray emitting globular clusters seen with the Fermi Large Area Telescope, arXiv:1003.3588.

[4] Fermi-LAT Collaboration, A. Abdo et. al., Fermi LAT observations of the Geminga pulsar, arXiv: 1007.1142.

[5] Fermi-LAT Collaboration, A. Abdo et. al., The First Fermi Large Area Telescope Catalog of Gamma-ray Pulsars, Astrophys.J.Suppl. 187 (2010) 460-494, [arXiv:0910.1608].

[6] E. Ferrara private communication (2010).

[7] P. G. Mezger, W. J. Duschl, and R. Zylka, The Galactic Center: a laboratory for AGN?, Astron. E Astrophys. Rev. 7 (1996) 289-388.

[8] M. P. Muno, F. K. Baganoff, M. W. Bautz, W. N. Brandt, P. S. Broos, E. D. Feigelson, G. P. Garmire, M. R. Morris, G. R. Ricker, and L. K. Townsley, A Deep Chandra Catalog of X-Ray Point Sources toward the Galactic Center, Astrophys. J. 589 (May, 2003) 225-241, [astro-ph/].

[9] G. Meylan, M. Mayor, A. Duquennoy, and P. Dubath, Central velocity dispersion in the globular cluster $\omega$ Centauri., Astron. \& Astrophys. 303 (Nov., 1995) 761-+. 
[10] D. Haggard, A. M. Cool, and M. B. Davies, A Chandra Study of the Galactic Globular Cluster Omega Centauri, Astrophys. J. 697 (May, 2009) 224-236, [arXiv:0902.2397]. 